\title{
Dynamic Changes in Liver Function Tests and Their Correlation with Illness Severity and Mortality in Patients with COVID-19: A Retrospective Cohort Study
}

Wei Xu*

Chenlu Huang (1D*

Ling Fei*

Qiang Li

Liang Chen

Department of Liver Diseases, Shanghai Public Health Clinical Center, Fudan University, Shanghai, 20I508, People's Republic of China

*These authors contributed equally to this work
Correspondence: Qiang Li; Liang Chen Email liqiang6660।@I63.com; chenliang@shphc.org.cn
Objective: To describe the longitudinal changes in liver function tests, and their association with illness severity and mortality in patients with COVID-19.

Methods: A retrospective cohort study of 1003 hospitalized patients with COVID-19 was conducted. Longitudinal liver function tests and clinical outcomes were analyzed.

Results: Abnormal liver function parameters were observed, both at admission (ALT 13.2\%, AST 8.5\%, ALP 2.0\%, GGT 7.4\%, LDH 37.6\%, TBIL 4.0\%, DBIL 7.8\%, Albumin 10.1\%) and peak hospitalization (ALT 29.4\%, AST 17.5\%, ALP 2.6\%, GGT 13.4\%, LDH 49.4\%, TBIL 10.1\%, DBIL 18.0\%, Albumin 30.6\%) in patients with COVID-19. Compared with non-severe patients, severe patients had markedly higher liver function parameters from baseline to 30 days after hospital admission. Abnormal ALT and LDH at hospital admission and some medications use (Hydroxychloroquine, Lopinavir/Ritonavir, and Traditional Chinese medicines) were associated with peak hospitalization ALT $>5 \times$ the upper limit unit of normal (ULN). On multivariate analysis, age $>60$ years, male, obesity, comorbidity, abnormal LDH and albumin at hospital admission and peak hospitalization were associated with progression to severe COVID-19 (OR $>1 ; p<0.05$ ). COX analysis revealed that ALT $>2$ ULN $(\mathrm{HR}=7.0, p=0.011)$, AST $>2 \operatorname{ULN}(\mathrm{HR}=34.7, p<$ $0.001)$, and TBIL $>2$ ULN $(\mathrm{HR}=54.6, p<0.001)$ were associated with a higher mortality.

Conclusion: Dynamic abnormalities of liver function parameters are common in hospitalized patients with COVID-19, and associated with illness severity and mortality.

Keywords: COVID-19, SARS-CoV-2, liver function parameters, liver injury, clinical outcomes

\section{Introduction}

Since November 2019, the outbreak of coronavirus disease 2019 (COVID-19), caused by severe acute respiratory syndrome coronavirus 2 (SARS-CoV-2), has influenced over 200 countries, areas or territories worldwide. ${ }^{1}$ The evidence that human-to-human transmission has been reported among close contacts of patients with COVID-19. ${ }^{2}$ Although considerable efforts have been made to reduce transmission, the overall upward trend of COVID-19 is continuing around the world. As of 17 January 2021, the outbreak of COVID-19 brings the cumulative numbers to over 93 million reported cases and over 2 million deaths globally. ${ }^{3}$

Although patients with COVID-19 present most commonly with respiratory symptoms, multiple extrapulmonary organ dysfunctions have also been reported. ${ }^{4}$ 
Previous studies have reported the prevalence of abnormal liver function parameters in patients with COVID-19, primarily alanine aminotransferase (ALT) (12.9-41.6\%) and aspartate aminotransferase (AST) (18.2-66.9\%). ${ }^{5-7}$ Furthermore, some studies have reported abnormal liver function parameters were associated with clinical outcomes of patients with COVID-19, including longer hospital stays, ${ }^{7}$ higher risk for severe COVID-19, ${ }^{6,8}$ and death. ${ }^{9}$

Different from previous reports, the severity rate and mortality rate of COVID-19 is relatively low in Shanghai, China, owing to the "Four Early Principle" (early detection, early diagnosis, early isolation, and early treatment). Data remain limited about the incidence and clinical value of liver injury in patients with COVID-19 from areas with low severity rate and mortality rate. In this cohort of 1003 hospitalized patients with COVID-19 in Shanghai, China, we aim to report the incidence of liver injury, describe the longitudinal changes in liver function parameters during the hospitalization, and evaluate the association between liver injury and illness severity and mortality.

\section{Methods}

\section{Participants}

A total of 1003 confirmed patients with COVID-19 admitted to Shanghai Public Health Clinical Center, Shanghai, China, between January 20th 2020 and October 20th 2020, were retrospectively analyzed. Patients with COVID- 19 were confirmed according to the positive results of SARS-CoV-2 RNA tests in nasopharyngeal or throat swab specimens using the polymerase chain reaction (PCR) method. ${ }^{10}$ Severe COVID-19 was diagnosed according to at least one of the following standards: $:^{10}$ (1) Respiratory frequency $\geq 30$ breath/min; (2) Resting oxygen saturation $\leq 93 \%$; (3) Oxygenation index $\leq 300 \mathrm{mmHg}$; (4) Mechanical ventilation; (5) shock; (6) Other organ failures and the intensive care unit (ICU) admission.

\section{SARS-CoV-2 RNA Extraction Method and PCR Protocol}

SARS-CoV-2 nucleic acids were detected using automatic magnetic extraction device and accompanying kit (BioGerm Medical Technology Co., Ltd, Shanghai, China) and screened with an RT-PCR kits (Bio-Germ Medical Technology Co., Ltd., Shanghai, China) with amplification targeting the ORF $1 \mathrm{a} / \mathrm{b}$ and $\mathrm{N}$ gene. The RT-PCR with $5 \mu \mathrm{L}$ RNA was used to target the nucleocapsid gene and open reading frame lab gene using a SARS-CoV-2 nucleic acid detection reagent (Bio-Germ Medical Technology Co., Ltd., Shanghai, China). The final reaction mixture concentration was $500 \mathrm{~nm}$ for primer, and $200 \mathrm{~nm}$ for probe, respectively. Conditions for the amplifications were $50^{\circ} \mathrm{C}$ for 15 minutes, $95^{\circ} \mathrm{C}$ for 3 minutes, followed by 45 cycles of $95^{\circ} \mathrm{C}$ for 15 seconds and $60^{\circ} \mathrm{C}$ for 30 seconds.

\section{Data Collection}

In this retrospective study, all data were extracted from the electronic medical records of Shanghai Public Health Clinical Center. Demographic data including age, sex, body mass index (BMI), and comorbidity were obtained. Clinical data including epidemiological histories, clinical manifestations, laboratory parameters, chest CT scans, hospital stays, and clinical outcomes were collected. Liver function tests including serum ALT, AST, alkaline phosphatase (ALP), gamma-glutamyl transpeptidase (GGT), lactate dehydrogenase (LDH), total bilirubin (TBIL), direct bilirubin (DBIL), and albumin, were performed using fully-auto-biochemistry-analysis-instruments (ARCHITECT C16000; ABBOTT LABORATORIES; SHANGHAI; CHINA).

\section{Liver Function Tests Abnormalities and Liver Injury Classifications}

Liver function test abnormalities were defined as the elevation of the following parameters in serum referring to Shanghai Public Health Clinical Center laboratory reference range standards: ALT $>44 \mathrm{U} / \mathrm{L}$, AST $>38 \mathrm{U} / \mathrm{L}$, ALP $>338 \mathrm{U} / \mathrm{L}, \mathrm{GGT}>73 \mathrm{U} / \mathrm{L}, \mathrm{LDH}>211 \mathrm{U} / \mathrm{L}, \mathrm{TBIL}>21$ umol/L, DBIL $>7 \mathrm{umol} / \mathrm{L}$, albumin $<38 \mathrm{~g} / \mathrm{L}$. As of now, the guidance or consensus on liver injury classifications are lacking for COVID-19 patients. However, as the magnitude of the liver function test elevations in our patients ranged from mild elevations to severe elevations. To describe the severity of liver injury, in this study, patients who had raised liver function parameters more than $5 \times$ the upper limit unit of normal (ULN) were classified as significant liver injury; patients who had raised liver function parameters 2-5 ULN were classified as moderate liver injury; and patients who had raised liver function parameters 1-2 ULN were classified as mild liver injury.

\section{Statistical Analysis}

Normally distributed data, non-normal distribution data, and categorical data were presented as mean \pm standard 
deviation, median (interquartile range, IQR), and frequency, respectively. The statistical differences were compared using the Student's $t$-test for normally distributed data, non-parametric Mann-Whitney-test for non-normal distribution data, and Chi-square test for categorical data. Clinical outcomes were modeled using liver function test results at admission and at their peak during hospitalization. Multivariate logistic regression analysis was used to adjust for age, gender, obesity, comorbidity, and liver function parameters. The Kaplan-Meier curves and estimates of survival data have become a familiar way of dealing with differing survival times (times-to-event). ${ }^{11}$ In this study, we performed the survival estimates using the Kaplan-Meier method, comparing the death rates according to the liver function parameters between the groups. All statistical analyses were performed in SPSS (version 16.0) and GraphPad Prism (version 6.0), and $p<0.05$ was considered statistically significant.

\section{Results}

\section{Baseline Characteristics of Patients}

Baseline characteristics of patients are summarized in Table 1. Of 1003 patients with COVID-19, the median age was 36 years (IQR, 25-51), 602 patients $(60.0 \%)$ were male, 288 patients $(28.7 \%)$ had obesity, and 183 patients $(18.2 \%)$ had comorbidity, mainly including hypertension (11.5\%) and diabetes mellitus (5.1\%). In this study, twenty-three patients had chronic liver diseases, including chronic hepatitis $B(n=15)$, alcoholic or nonalcoholic fatty liver disease $(n=9)$, and autoimmune liver disease $(n=1)$. Two patients had both chronic hepatitis B and fatty liver disease. Twenty-four patients had chronic heart diseases, including coronary artery disease $(n=20)$, chronic cardiac dysfunction $(n=4)$, and cardiomyopathy $(n=3)$. Three patients had both coronary artery disease and chronic cardiac dysfunction. Sixteen patients had chronic pulmonary diseases, including asthma $(\mathrm{n}=10)$, chronic obstructive pulmonary disease $(n=4)$, and interstitial pneumonia $(n=2)$.

Table I Baseline Characteristics of the Study Population

\begin{tabular}{|c|c|c|c|c|}
\hline & Total & Non-Severe Group & Severe Group & p-value \\
\hline Number & 1003 & 968 & 35 & - \\
\hline Age (years) & $36(25-5 I)$ & $35(25-50)$ & $64(49-74)$ & $<0.001$ \\
\hline Male gender, n (\%) & $602(60.0 \%)$ & 574 (59.3\%) & $28(80 \%)$ & 0.014 \\
\hline BMI (kg/m2) & $26.3 \pm 6.5$ & $26.2 \pm 6.2$ & $27.9 \pm 7.8$ & $<0.001$ \\
\hline Obesity, n (\%) & 288 (28.7\%) & $270(27.9 \%)$ & $18(5 \mid .4 \%)$ & 0.003 \\
\hline $\begin{array}{l}\text { Comorbidity, n (\%) } \\
\text { Hypertension } \\
\text { Diabetes mellitus } \\
\text { CHD } \\
\text { CLD } \\
\text { CPD } \\
\text { CKD }\end{array}$ & $\begin{array}{l}\text { I83 (I8.2\%) } \\
115(11.5 \%) \\
51(5.1 \%) \\
24(2.4 \%) \\
23(2.3 \%) \\
16(1.6 \%) \\
5(0.5 \%)\end{array}$ & $\begin{array}{l}159(16.4 \%) \\
97(10.0 \%) \\
44(4.5 \%) \\
18(1.9 \%) \\
22(2.3 \%) \\
12(1.2 \%) \\
4(0.4 \%)\end{array}$ & $\begin{array}{l}24(68.6 \%) \\
18(51.4 \%) \\
7(20 \%) \\
6(17.1 \%) \\
1(2.9 \%) \\
4(11.4 \%) \\
I(2.9 \%)\end{array}$ & $\begin{array}{l}<0.001 \\
<0.001 \\
<0.001 \\
<0.001 \\
0.821 \\
<0.001 \\
0.044\end{array}$ \\
\hline $\begin{array}{l}\text { Antiviral therapy } \\
\text { TCM } \\
\text { Hydroxychloroquine } \\
\text { Lopinavir/Ritonavir }\end{array}$ & $\begin{array}{l}5 \mathrm{II}(50.9 \%) \\
278(27.7 \%) \\
124(12.4 \%)\end{array}$ & $\begin{array}{l}505(52.2 \%) \\
274(28.3 \%) \\
111(11.5 \%)\end{array}$ & $\begin{array}{l}6(17.1 \%) \\
4(11.4 \%) \\
13(37.1 \%)\end{array}$ & $\begin{array}{l}<0.001 \\
0.028 \\
<0.001\end{array}$ \\
\hline $\begin{array}{l}\text { Laboratory findings } \\
\text { WBC }\left(10^{9} / \mathrm{L}\right) \\
\text { Lymphocyte }\left(10^{9} / \mathrm{L}\right) \\
\text { Platelet }\left(10^{9} / \mathrm{L}\right) \\
\text { PCT }(\mathrm{ng} / \mathrm{mL}) \\
\text { CRP }(\mathrm{mg} / \mathrm{L}) \\
\text { ESR }(\mathrm{mm} / \mathrm{h})\end{array}$ & $\begin{array}{l}5.7(4.4-7.0) \\
1.5(1.1-2.0) \\
217(172-262) \\
0.05(0.02-0.10) \\
0.5(0.5-6.0) \\
27(10-53)\end{array}$ & $\begin{array}{l}5.7(4.5-7.0) \\
1.5(1.1-2.0) \\
220(175-265) \\
0.05(0.02-0.10) \\
0.5(0.5-4.7) \\
26(10-50)\end{array}$ & $\begin{array}{l}5.9(3.6-7.9) \\
0.7(0.5-1.0) \\
172(121-209) \\
0.10(0.06-0.15) \\
37.6(12.9-78.7) \\
52(38-89)\end{array}$ & $\begin{array}{l}0.668 \\
<0.001 \\
<0.001 \\
<0.001 \\
<0.001 \\
<0.001\end{array}$ \\
\hline
\end{tabular}

Note: The $p$ values indicate differences between severe group and non-severe group.

Abbreviations: CHD, chronic heart diseases; CLD, chronic liver diseases; CPD, chronic pulmonary diseases; CKD, chronic kidney diseases; TCM, Traditional Chinese medicines; WBC, white blood count; PCT, procalcitonin; CRP, C-reactive protein; ESR, erythrocyte sedimentation rate. 
Five patients had chronic kidney diseases, including chronic renal dysfunction $(\mathrm{n}=4)$ and nephrotic syndrome $(\mathrm{n}=1)$. The median levels of white blood count (WBC), lymphocyte, platelet, procalcitonin (PCT), C-reactive protein (CRP), and erythrocyte sedimentation rate (ESR) were $5.7 \times 10^{9} / \mathrm{L}$ (IQR, $4.4-7.0), \quad 1.5 \times 10^{9} / \mathrm{L} \quad(\mathrm{IQR}, \quad 1.1-2.0), 217 \times 10^{9} / \mathrm{L} \quad$ (IQR, 172-262), $\quad 0.05 \quad \mathrm{ng} / \mathrm{mL} \quad(\mathrm{IQR}, \quad 0.02-0.10), \quad 0.5 \quad \mathrm{mg} / \mathrm{L}$ (IQR, 0.5-6.0), and $27 \mathrm{~mm} / \mathrm{h}$ (IQR, 10-53), respectively.

Severe patients with COVID-19 had higher age (median, 64 vs 35 years, $p<0.001$ ) and BMI (mean, 27.9 vs $26.2 \mathrm{~kg} /$ $\mathrm{m} 2, p<0.001$ ), more common male gender ( $80 \%$ vs $59.3 \%$, $p=0.014)$, obesity ( $51.4 \%$ vs $27.9 \%, p=0.003)$, and comorbidity $(68.6 \%$ vs $16.4 \%, p<0.001)$ than non-severe patients (Table 1). Compared with non-severe patients with COVID19 , severe patients had significantly higher PCT ( 0.10 vs 0.05 $\mathrm{ng} / \mathrm{mL}, p<0.001)$, CRP (37.6 vs $0.5 \mathrm{mg} / \mathrm{L}, p<0.001$ ), ESR (52 vs $26 \mathrm{~mm} / \mathrm{h}, p<0.001$ ), but significantly lower lymphocyte count $\left(0.7\right.$ vs $\left.1.5 \times 10^{9} / \mathrm{L}, p<0.001\right)$ and platelet count $\left(172\right.$ vs $\left.220 \times 10^{9} / \mathrm{L}, p<0.001\right)$ (Table 1$)$.

\section{Antiviral Medications Use During Hospitalization}

Antiviral medications were assessed, including Traditional Chinese medicines (TCM) (50.9\%), hydroxychloroquine (27.7\%), and lopinavir/ritonavir (12.4\%). In this retrospective study, TCM included Ganlu Xiaodu Micropills,
Yinqiao Powder, Xiangsu Powder, Shengjiang Powder, Agastache, Pinellia and Poria Decoction, Sanren Decoction, Maxing Shigan Decoction, Little Bupleurum Decoction, Qingfeipaidu decoction, Baihu Decoction, Dachengqi Decoction, LungCleansing and Detoxifying Decoction, Jinhua Qinggan Granule, Lianhua Qingwen Capsule, Huoxiang Zhengqi capsules, Shufengjiedu capsules, Huashibaidu Formula, Xuanfeibaidu Granule, and Xuebijing Injection.

\section{Liver Function Parameters of 1003}

\section{Patients on Hospital Admission}

Liver function parameters of 1003 patients on hospital admission are summarized in Table 2. The median levels of ALT, AST, ALP, GGT, LDH, TBIL, DBIL, and albumin were $20 \mathrm{U} / \mathrm{L}$ (IQR, 14-31), $20 \mathrm{U} / \mathrm{L}$ (IQR, 17-26), 75 U/L (IQR, 55-193), 21 U/L (IQR, 14-36), 198 U/L (IQR, 172-232), 8.4 umol/L (IQR, 6.5-11.3), 3.4 umol/L (IQR, 2.3-4.6), and $45 \mathrm{~g} / \mathrm{L}$ (IQR, 41-47), respectively. Severe patients had significantly higher levels of ALT (26 vs 20 $\mathrm{U} / \mathrm{L}, p=0.015$ ), AST (31 vs $20 \mathrm{U} / \mathrm{L}, p<0.001)$, GGT (30 vs $21 \mathrm{U} / \mathrm{L}, p<0.001)$, LDH (334 vs $197 \mathrm{U} / \mathrm{L}, p<0.001)$, TBIL ( 10.2 vs $8.3 \mathrm{umol} / \mathrm{L}, p=0.026$ ), DBIL ( 4.9 vs $3.3 \mathrm{umol} / \mathrm{L}$, $p<0.001$ ), but significantly lower albumin ( 37 vs $45 \mathrm{~g} / \mathrm{L}$, $p<0.001)$ than non-severe patients. Abnormal AST $(42.9 \%$ vs $7.2 \%, p<0.001)$, $\mathrm{LDH}(88.6 \%$ vs $35.7 \%, p<0.001)$,

Table 2 Liver Function Tests of 1003 Patients with COVID-19 on Hospital Admission

\begin{tabular}{|c|c|c|c|c|}
\hline Characteristic & Total & Non-Severe & Severe & p-value \\
\hline Number & 1003 & 968 & 35 & - \\
\hline $\operatorname{ALT}(\mathrm{U} / \mathrm{L})$ & $20(|4-3|)$ & $20(|4-3|)$ & $26(16-36)$ & 0.015 \\
\hline ALT, abnormal (> $44 \mathrm{U} / \mathrm{L})$ & 132 (13.2\%) & 125 (12.9\%) & $7(20 \%)$ & 0.223 \\
\hline AST (U/L) & $20(17-26)$ & $20(17-26)$ & $31(24-5 I)$ & $<0.001$ \\
\hline AST, abnormal (> $38 \mathrm{U} / \mathrm{L})$ & $85(8.5 \%)$ & $70(7.2 \%)$ & $15(42.9 \%)$ & $<0.001$ \\
\hline $\operatorname{ALP}(\mathrm{U} / \mathrm{L})$ & $75(55-193)$ & $75(55-196)$ & $69(49-177)$ & 0.486 \\
\hline ALP, abnormal (> $338 \mathrm{U} / \mathrm{L})$ & $20(2.0 \%)$ & $20(2.1 \%)$ & 0 & 0.930 \\
\hline GGT (U/L) & $21(14-36)$ & $21(14-35)$ & $30(2 I-60)$ & $<0.001$ \\
\hline GGT, abnormal (> $73 \mathrm{U} / \mathrm{L})$ & 74 (7.4\%) & $69(7.1 \%)$ & $5(14.3 \%)$ & 0.112 \\
\hline $\mathrm{LDH}(\mathrm{U} / \mathrm{L})$ & $198(172-232)$ & $197(|7|-229)$ & $334(264-452)$ & $<0.001$ \\
\hline LDH, abnormal (> 2II U/L) & 377 (37.6\%) & 346 (35.7\%) & $31(88.6 \%)$ & $<0.001$ \\
\hline TBIL (umol/L) & $8.4(6.5-11.3)$ & $8.3(6.5-11.2)$ & $10.2(7.9-14.9)$ & 0.026 \\
\hline TBIL, abnormal (> 2I umol/L) & $40(4.0 \%)$ & $38(3.9 \%)$ & $2(5.7 \%)$ & 0.959 \\
\hline DBIL (umol/L) & $3.4(2.3-4.6)$ & $3.3(2.3-4.5)$ & $4.9(3.7-7.0)$ & $<0.001$ \\
\hline DBIL, abnormal (> 7 umol/L) & $78(7.8 \%)$ & $70(7.2 \%)$ & $8(22.9 \%)$ & $<0.001$ \\
\hline Albumin $(g / L)$ & $45(4 \mid-47)$ & $45(42-47)$ & $37(33-40)$ & $<0.001$ \\
\hline Albumin, abnormal (<38 g/L) & $101(10.1 \%)$ & $83(8.6 \%)$ & 18 (5I.4\%) & $<0.001$ \\
\hline
\end{tabular}

Notes: The $p$ values indicate differences between severe group and non-severe group. $p<0.05$ was considered statistically significant.

Abbreviations: ALT, alanine aminotransferase; AST, aspartate aminotransferase; ALP, alkaline phosphatase; GGT, gamma-glutamyl transpeptidase; LDH, lactate dehydrogenase; TBIL, total bilirubin; DBIL, direct bilirubin. 
DBIL (22.9\% vs $7.2 \%, p<0.001)$, and albumin $(51.4 \%$ vs $8.6 \%, p<0.001)$ were commonly observed in severe patients, compared with non-severe patients.

\section{Hospital Admission vs Peak Hospitalization Liver Function Parameters in 1003 Patients}

Hospital admission vs peak hospitalization liver function tests in 1003 patients with COVID-19 are shown in Table 3. Abnormal liver function parameters were observed at admission (ALT 13.2\%, AST 8.5\%, ALP 2.0\%, GGT 7.4\%, LDH
$37.6 \%$, TBIL 4.0\%, DBIL 7.8\%, albumin 10.1\%) and peak hospitalization (ALT 29.4\%, AST 17.5\%, ALP 2.6\%, GGT 13.4\%, LDH 49.4\%, TBIL 10.1\%, DBIL 18.0\%, albumin $30.6 \%$ ) in hospitalized patients with COVID-19. Most patients with abnormal liver function parameters had minimal elevations 1-2 ULN at admission (ALT 84.8\%, AST 84.7\%, ALP 85\%, GGT 78.4\%, LDH 94.2\%, TBIL 95.0\%, DBIL 93.6\%), as well as peak hospitalization (ALT 68.1\%, AST 77.8\%, ALP 88.5\%, GGT 72.4\%, LDH 89.1\%, TBIL 90.1\%, DBIL $86.7 \%$ ). The significant elevations of liver function tests $(>5$ ULN) were rarely observed (ALT 4.7\%, AST 2.3\%, ALP 0, GGT 3.0\%, LDH 0, TBIL 3.0\%, DBIL 0.6\%) during

Table 3 Hospital Admission vs Peak Hospitalization Liver Tests in 1003 Patients with COVID-19

\begin{tabular}{|c|c|c|c|}
\hline & & Hospital Admission & Peak Hospitalization \\
\hline \multirow[t]{4}{*}{ ALT (U/L) } & Abnormal (> 44 U/L) & $132(13.2 \%)$ & 295 (29.4\%) \\
\hline & I-2 ULN & II 2 (84.8\%) & 201 (68.1\%) \\
\hline & 2-5 ULN & 19 (14.4\%) & $80(27.1 \%)$ \\
\hline & $>5$ ULN & I (0.8\%) & $14(4.7 \%)$ \\
\hline \multirow[t]{4}{*}{ AST (U/L) } & Abnormal (> 38 U/L) & 85 (8.5\%) & 176 (I7.5\%) \\
\hline & I-2 ULN & 72 (84.7\%) & I 37 (77.8\%) \\
\hline & 2-5 ULN & 12 (14.1\%) & 35 (19.9\%) \\
\hline & $>5$ ULN & $\mathrm{I}(\mathrm{I} .2 \%)$ & $4(2.3 \%)$ \\
\hline \multirow[t]{4}{*}{ ALP (U/L) } & Abnormal (> $338 \mathrm{U} / \mathrm{L})$ & $20(2.0 \%)$ & $26(2.6 \%)$ \\
\hline & I-2 ULN & 17 (85\%) & 23 (88.5\%) \\
\hline & 2-5 ULN & $3(15 \%)$ & 3 (II.5\%) \\
\hline & $>5$ ULN & 0 & 0 \\
\hline \multirow[t]{4}{*}{ GGT (U/L) } & Abnormal (> 73 U/L) & 74 (7.4\%) & I34 (I3.4\%) \\
\hline & I-2 ULN & 58 (78.4\%) & 97 (72.4\%) \\
\hline & 2-5 ULN & 14 (I8.9\%) & $33(24.6 \%)$ \\
\hline & $>5$ ULN & $2(2.7 \%)$ & $4(3.0 \%)$ \\
\hline \multirow[t]{4}{*}{ LDH (U/L) } & Abnormal (> 2II U/L) & 377 (37.6\%) & 495 (49.4\%) \\
\hline & I-2 ULN & 355 (94.2\%) & 44। (89.1\%) \\
\hline & $2-5$ ULN & 22 (5.8\%) & 54 (10.9\%) \\
\hline & $>5$ ULN & 0 & 0 \\
\hline \multirow[t]{4}{*}{ TBIL (umol/L) } & Abnormal (> 2l umol/L) & $40(4.0 \%)$ & 101 (10.1\%) \\
\hline & I-2 ULN & 38 (95\%) & 91 (90.1\%) \\
\hline & 2-5 ULN & I (2.5\%) & 7 (6.9\%) \\
\hline & $>5$ ULN & I (2.5\%) & $3(3.0 \%)$ \\
\hline \multirow[t]{4}{*}{ DBIL (umol/L) } & Abnormal (> 7 umol/L) & 78 (7.8\%) & 180 (I8.0\%) \\
\hline & I-2 ULN & 73 (93.6\%) & I56 (86.7\%) \\
\hline & 2-5 ULN & $5(6.4 \%)$ & $23(12.8 \%)$ \\
\hline & $>5$ ULN & 0 & I (0.6\%) \\
\hline \multirow[t]{3}{*}{ Albumin $(g / L)$} & Abnormal (< 38 g/L) & 101 (10.1\%) & 307 (30.6\%) \\
\hline & 32-38 (g/L) & 91 (90.1\%) & 238 (77.5\%) \\
\hline & $<32(\mathrm{~g} / \mathrm{L})$ & $10(9.9 \%)$ & $69(22.5 \%)$ \\
\hline
\end{tabular}

Abbreviations: ALT, alanine aminotransferase; AST, aspartate aminotransferase; ALP, alkaline phosphatase; GGT, gamma-glutamyl transpeptidase; LDH, lactate dehydrogenase; TBIL, total bilirubin; DBIL, direct bilirubin; ULN, upper limit of normal. 
hospitalization. Most patients with abnormal liver function parameters had minimal reduction of albumin $(32-38 \mathrm{~g} / \mathrm{L})$ at admission (90.1\%), as well as peak hospitalization (77.5\%), and a small subset of patients had significant reduction of albumin $(<32 \mathrm{~g} / \mathrm{L})$ at admission $(9.9 \%)$, as well as peak hospitalization $(22.5 \%)$.

\section{Predictors of Peak Hospitalization ALT $>5$ ULN}

Predictors of ALT $>5$ ULN at time of peak liver test value during hospitalization are summarized in Table 4, including abnormal ALT and LDH on hospital admission, and medications use (Hydroxychloroquine, Lopinavir/Ritonavir, and TCM) during hospitalization. Compared to patients with $\mathrm{ALT} \leq 5 \mathrm{ULN}$, those with ALT $>5 \mathrm{ULN}$ had more common abnormal ALT $(35.7 \%$ vs $12.8 \%, p=0.012)$ and $\mathrm{LDH}$ (78.6\% vs $31.0 \%, p=0.001)$ on hospital admission, and more Hydroxychloroquine $(57.1 \%$ vs $27.3 \%, p=0.013)$, Lopinavir/Ritonavir (42.9\% vs $11.9 \%, p<0.001)$, and TCM $(78.6 \%$ vs $50.6 \%, p=0.037)$ use.

\section{Association Between Liver Function Parameters and Clinical Outcomes}

The association between liver function parameters and clinical outcomes is shown in Table 5. On multivariate analysis, age $>60$ years, male gender, BMI $>30 \mathrm{~kg} / \mathrm{m} 2$, comorbidity, abnormal LDH and albumin on hospital admission, and abnormal peak hospitalization LDH and albumin were associated with progression to severe COVID-19 (OR $>1 ; p<0.05)$. The dynamic profile of liver function parameters in patients by severity of COVID-19 is illustrated in Figure 1. Severe COVID-19 patients had markedly higher levels of ALT, AST, GGT, LDH, TBIL, DBIL, but significantly lower levels of albumin than non-severe patients from baseline to 30 days after admission $(p<0.05)$ (Figure 1$)$. The peak of ALT, LDH, TBIL, DBIL value, and the trough of albumin was observed on 6-10 day of hospitalization. The peak of ALP and GGT value was observed on 11-15 day of hospitalization (Figure 1).

On multivariate analysis, age $>60$ years $(\mathrm{OR}=6.44$; 95\% CI 2.24-14.77; $p<0.005)$, BMI $>30 \mathrm{~kg} / \mathrm{m} 2$ $(\mathrm{OR}=1.78 ; 95 \%$ CI $1.23-4.35 ; p=0.024)$, comorbidity $(\mathrm{OR}=6.74 ; 95 \%$ CI 2.93-21.85; $p<0.001)$, and abnormal peak hospitalization ALT $(\mathrm{OR}=3.37$; 95\% CI 1.25-8.16; $p=0.008)$, AST $(\mathrm{OR}=4.82 ; 95 \%$ CI 1.28-16.16; $p<0.001)$, and TBIL $(\mathrm{OR}=5.65 ; 95 \%$ CI 1.87-18.20; $p<0.001)$ were associated with death. Kaplan-Meier curves for cumulative rate of death in patients with different level of ALT (a), AST (b), and TBIL (c) are illustrated in Figure 2. ALT $>2$ ULN $(\mathrm{HR}=7.0 ; \mathrm{CI} \%=1.6-31.4 ; p=0.011), \mathrm{AST}>2 \mathrm{ULN}$ $(\mathrm{HR}=34.7 ; \mathrm{CI} \%=7.8-155.3 ; p<0.001)$, and TBIL $>2$ ULN $(\mathrm{HR}=54.6 ; \mathrm{CI} \%=6.6-453.8 ; p<0.001)$ were associated with a higher mortality.

\section{Discussion}

Although COVID-19 is well known for causing respiratory symptoms, it can also cause extrapulmonary manifestations, including hepatocellular injury. ${ }^{12}$ In this study of

Table 4 Predictors of ALT > 5 ULN at Time of Peak Liver Test Value During Hospitalization

\begin{tabular}{|c|c|c|c|}
\hline \multirow[t]{2}{*}{ Characteristic } & \multicolumn{3}{|c|}{ ALT at Time of Peak Liver Test Value } \\
\hline & $<5$ ULN (n=989) & $>5$ ULN $(n=14)$ & $\mathrm{p}$ value \\
\hline Age (years) & $36(25-5 I)$ & $30(26-55)$ & 0.707 \\
\hline Male gender, $\mathrm{n}(\%)$ & $593(60 \%)$ & $9(64.3 \%)$ & 0.743 \\
\hline BMI (kg/m2) & $26.2 \pm 6.4$ & $27.0 \pm 6.6$ & 0.889 \\
\hline Diabetes mellitus, n (\%) & $50(5.1 \%)$ & $\mathrm{I}(7.1 \%)$ & 0.716 \\
\hline Hypertension, n (\%) & 114 (II.5\%) & I (7.1\%) & 0.609 \\
\hline Chronic liver disease, n (\%) & $23(2.3 \%)$ & 0 & 0.566 \\
\hline Abnormal ALT on hospital admission, $n$ (\%) & 127 (I2.8\%) & $5(35.7 \%)$ & 0.012 \\
\hline Abnormal AST on hospital admission, $\mathrm{n}(\%)$ & $82(8.3 \%)$ & $3(21.4 \%)$ & 0.08 \\
\hline Abnormal GGT on hospital admission, $\mathrm{n}$ (\%) & $73(7.4 \%)$ & I (7.l\%) & 0.973 \\
\hline Abnormal LDH on hospital admission, $n$ (\%) & $366(37.0 \%)$ & II (78.6\%) & 0.001 \\
\hline Abnormal TBIL on hospital admission, $\mathrm{n}$ (\%) & $40(4.0 \%)$ & 0 & 0.443 \\
\hline Hydroxychloroquine use, n (\%) & $270(27.3 \%)$ & $8(57.1 \%)$ & 0.013 \\
\hline Lopinavir/Ritonavir use, n (\%) & $118(11.9 \%)$ & $6(42.9 \%)$ & $<0.001$ \\
\hline Traditional Chinese Medicine use, $n(\%)$ & $500(50.6 \%)$ & II (78.6\%) & 0.037 \\
\hline
\end{tabular}

Abbreviations: ALT, alanine aminotransferase; ULN, upper limit of normal; AST, aspartate aminotransferase; GGT, gamma-glutamyl transpeptidase; LDH, lactate dehydrogenase; TBIL, total bilirubin. 
Table 5 Association Between Admission and Peak Hospitalization Liver Tests and Clinical Outcomes

\begin{tabular}{|c|c|c|c|c|}
\hline & $\begin{array}{l}\text { Severe COVID-19 } \\
\text { (Multivariate Model) }\end{array}$ & & $\begin{array}{l}\text { Death } \\
\text { (Multivariate Model) }\end{array}$ & \\
\hline & OR $(90 \% \mathrm{Cl})$ & p-value & OR $(90 \% \mathrm{Cl})$ & p-value \\
\hline Age $>60$ years & $4.02(1.58-10.20)$ & 0.003 & $6.44(2.24-14.77)$ & 0.005 \\
\hline Male gender & $2.7 \mid(1.27-8.79)$ & 0.019 & $1.46(0.28-6.35)$ & 0.368 \\
\hline $\mathrm{BMI}>30 \mathrm{~kg} / \mathrm{m} 2$ & $3.46(1.32-9.43)$ & 0.012 & $1.78(1.23-4.35)$ & 0.024 \\
\hline Comorbidity & $6.08(2.45-15.10)$ & $<0.001$ & $6.74(2.93-21.85)$ & $<0.001$ \\
\hline \multicolumn{5}{|l|}{ Hospital Admission } \\
\hline Abnormal ALT & $0.37(0.07-1.90)$ & 0.234 & $0.99(0.04-26.30)$ & 0.994 \\
\hline Abnormal AST & $3.01(0.70-13.03)$ & 0.141 & $1.50(0.16-14.47)$ & 0.725 \\
\hline Abnormal ALP & $0.22(0.01-5.86)$ & 0.218 & $0.85(0.12-18.95)$ & 0.799 \\
\hline Abnormal GGT & $0.67(0.12-1.19)$ & 0.951 & $0.58(0.07-4.55)$ & 0.601 \\
\hline Abnormal LDH & $3.36(1.41-8.78)$ & 0.002 & $2.11(0.29-15.48)$ & 0.464 \\
\hline Abnormal TBIL & $0.49(0.01-17.06)$ & 0.696 & $1.02(0.07-14.73)$ & 0.986 \\
\hline Abnormal DBIL & $1.85(0.14-6.31)$ & 0.325 & $4.62(0.45-47.39)$ & 0.197 \\
\hline Abnormal Albumin & $2.45(I .16-7.5 \mathrm{I})$ & 0.026 & $4.74(0.70-31.95)$ & 0.110 \\
\hline \multicolumn{5}{|l|}{ Peak Hospitalization } \\
\hline Abnormal ALT & $2.28(0.96-6.46)$ & 0.139 & $3.37(1.25-8.16)$ & 0.008 \\
\hline Abnormal AST & $3.92(0.56-11.25)$ & 0.141 & $4.82(1.28-16.16)$ & $<0.001$ \\
\hline Abnormal ALP & $0.29(0.0 \mathrm{I}-7.2 \mathrm{I})$ & 0.453 & $1.45(0.07-30.66)$ & 0.811 \\
\hline Abnormal GGT & $2.15(0.98-7.86)$ & 0.088 & $3.79(0.60-57.87)$ & 0.117 \\
\hline Abnormal LDH & $3.84(1.67-9.88)$ & $<0.001$ & $0.89(0.01-47.85)$ & 0.558 \\
\hline Abnormal TBIL & $0.21(0.03-1.66)$ & 0.139 & $5.65(1.87-18.20)$ & $<0.001$ \\
\hline Abnormal DBIL & $0.49(0.01-17.06)$ & 0.696 & $1.39(0.05-35.27)$ & 0.806 \\
\hline Abnormal Albumin & $3.94(1.36-11.43)$ & 0.002 & $2.16(0.87-5.59)$ & 0.747 \\
\hline
\end{tabular}

Abbreviations: OR, odds ratio; $\mathrm{Cl}$, confidence interval; ALT, alanine aminotransferase; AST, aspartate aminotransferase; ALP, alkaline phosphatase; GGT, gamma-glutamyl transpeptidase; LDH, lactate dehydrogenase; TBIL, total bilirubin; DBIL, direct bilirubin.

1003 patients with COVID-19, ALT and AST abnormalities were observed in $13.2 \%$ and $8.5 \%$ of patients at admission, respectively, and in $29.4 \%$ and $17.5 \%$ of patients at peak hospitalization, respectively. Based on a meta-analysis, the pooled prevalence estimates of elevated liver function abnormalities in China were as follows: ALT $15.0 \%$ and AST $15.0 \%{ }^{13}$ However, some studies from America showed higher prevalence ranging between $40-50.6 \%$ in cohorts ranging from 116 to 2780 patients. $^{14-16}$ Obviously, abnormal liver function parameters are less common in Chinese patients than that reported in the U.S. ${ }^{5,13,15,16}$ The differences in baseline factors (chronic liver diseases, obesity, alcohol consumption) and hospital management (antiviral medication use) may potentially account for some of this disparity. Moreover, the different laboratory references of liver function parameters in different health-care systems might lead to the different definitions of liver injury, which may be one of the reasons for the disparity in the prevalence of liver injury between Chinese patients and the US patients. For example, the ULN of ALT ranges from $40 \mathrm{U} / \mathrm{L}$ to 50 $\mathrm{U} / \mathrm{L}$ in the studies from China, ${ }^{6,8,9}$ but ranges from $33 \mathrm{U} / \mathrm{L}$ to $50 \mathrm{U} / \mathrm{L}$ in the studies from the US. ${ }^{5,16,17}$

This study showed that the pattern of abnormal liver function tests is predominantly hepatocellular (at admission: ALT 13.2\%, AST 8.5\%; at peak hospitalization: ALT $29.4 \%$, AST $17.5 \%$ ) rather than cholestatic, although less common elevations in ALP (2.0\% at admission, and $2.6 \%$ at peak hospitalization), GGT (7.4\% at admission, and $13.4 \%$ at peak hospitalization), and TBIL (4.0\% at admission, and $10.1 \%$ at peak hospitalization) can be observed. Given that angiotensin converting enzyme-2 (ACE2), the entry receptor for SARS-CoV-2, is much more heavily expressed in cholangiocytes than in hepatocytes, ${ }^{18}$ therefore our findings suggest that the direct cytopathic effect of the SARS-CoV-2 may not be the main mechanism of COVID-19-related liver damage. Hepatic dysfunction in COVID-19 could be related to an uncontrolled immune 


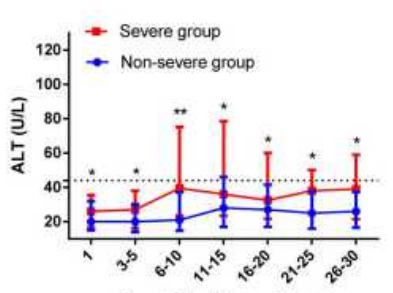

Days After Disease Onset

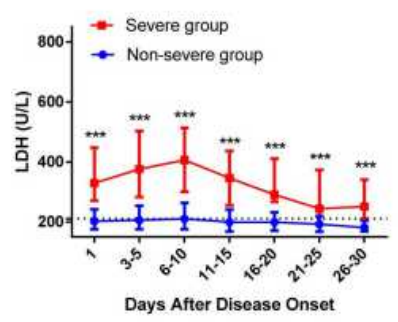

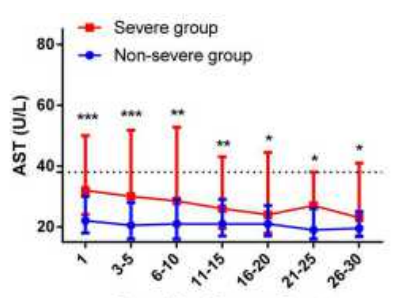

Days After Disease Onset

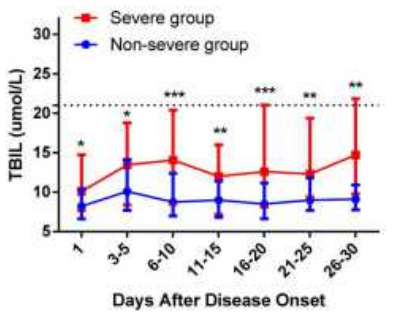

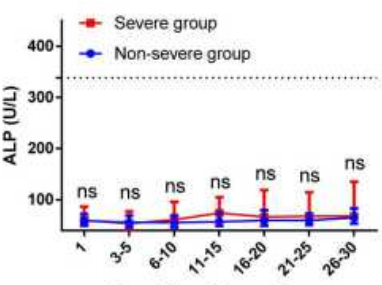

Days After Disease Onset

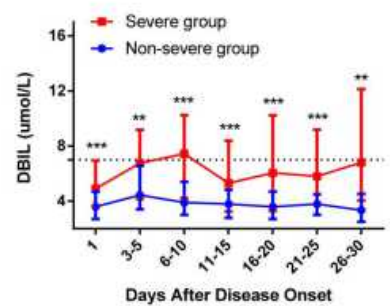

Days After Disease Onset

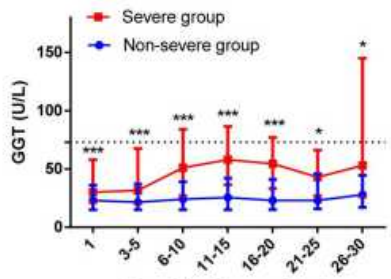

Days After Disease Onset

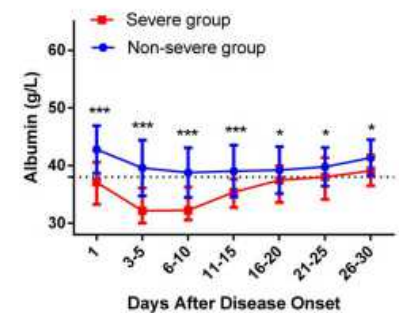

26-30

19

$\begin{array}{llllcccc}\text { Time (days) } & 1 & 3-5 & 6-10 & 11-15 & 16-20 & 21-25 & 26-30 \\ \text { Severe group (n) } & 35 & 33 & 33 & 31 & 29 & 23 & 19 \\ \text { Non-severe group (n) } & 968 & 720 & 683 & 417 & 187 & 91 & 35\end{array}$

Figure I Dynamic profile of liver function tests in patients by severity of COVID-19. The liver function tests in non-severe group (blue line) and severe group (red line) were analyzed at different time points after hospital admission. The liver function tests are shown using median and IQR. “*” means $p<0.05$; “**” means $p<0.0 \mathrm{I}$; “***” means $p<$ 0.001 . Severe group had markedly higher levels of ALT, AST, GGT, LDH, TBIL, DBIL, but significantly lower levels of albumin compared with non-severe group from baseline to 30 days after admission $(p<0.05)$.
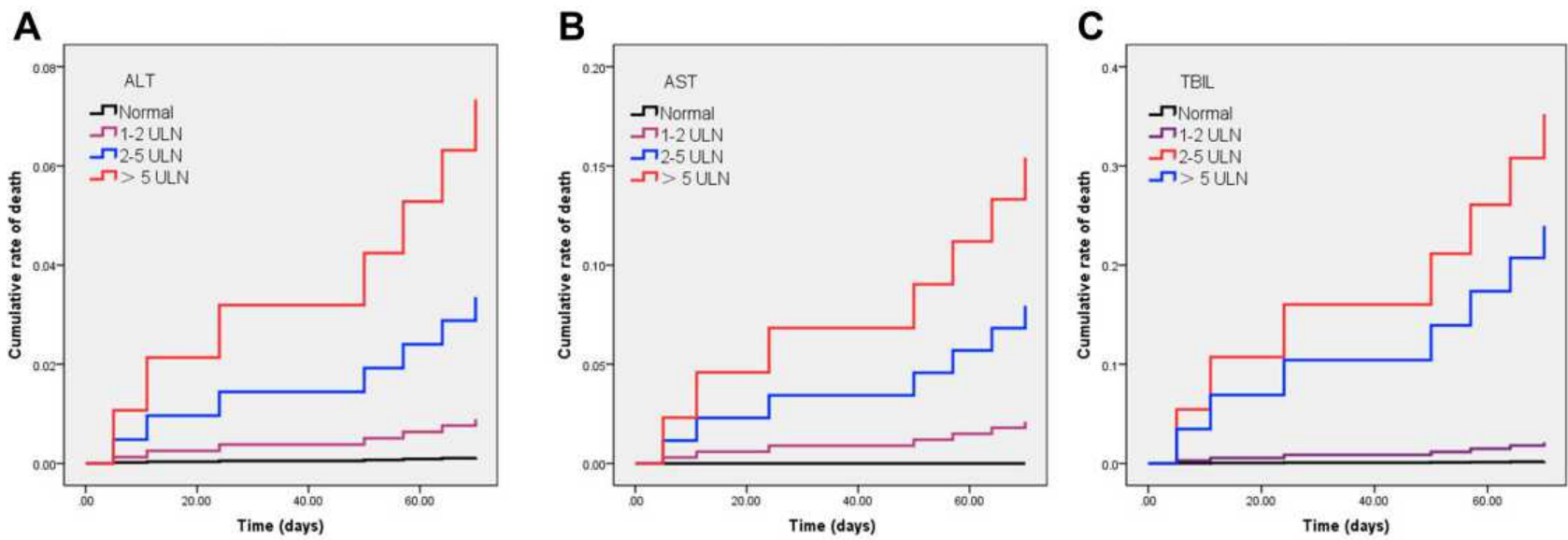

Figure 2 Kaplan-Meier curves for cumulative rate of death during hospitalization in patients with different level of ALT (A), AST (B), and TBIL (C). ALT > 2 ULN (HR=7.0; $\mathrm{Cl} \%=1.6-31.4 ; p=0.0 \mathrm{II})$, AST $>2 \mathrm{ULN}(\mathrm{HR}=34.7 ; \mathrm{Cl} \%=7.8-155.3 ; p<0.00 \mathrm{I})$, and TBIL $>2 \mathrm{ULN}(\mathrm{HR}=54.6 ; \mathrm{Cl} \%=6.6-453.8 ; p<0.00 \mathrm{I})$ were associated with a higher mortality.

reaction, sepsis or drug-induced liver injury, besides the direct cytopathic effect of the virus. ${ }^{19}$

Abnormal liver parameters are usually minimally elevated, although some significant abnormal liver function parameters ( $>5$ ULN) (ALT 4.7\%, AST 2.3\%, GGT 3.0\%, TBIL 3.0\%, DBIL 0.6\%) may be observed at peak hospitalization. The current results are consistent with prior observations. $^{5,6}$ An American study reported that only $5.9 \%$ and $6.0 \%$ of ALT and AST elevations, respectively, were beyond 5 ULN at hospital admission, and 20.6\% and $16.6 \%$ of ALT and AST elevations, respectively, were beyond 5 ULN at peak hospitalization. ${ }^{5}$ A Hong Kong study of 1040 COVID-19 patients reported that only $4.9 \%$ and $1.3 \%$ of ALT and AST elevations, respectively, were beyond 5 ULN during hospitalization. ${ }^{20}$

This study shows an association between antiviral medications use (Hydroxychloroquine, Lopinavir/ Ritonavir, and TCM) and peak hospitalization ALT > 5 
ULN in patients with COVID-19. Previous studies also showed that the use of certain drugs showed an association with the progression of liver damage in patients with COVID-19. 5,7,20 An American study reported that Hydroxychloroquine and Lopinavir/Ritonavir use was the predictor of peak hospitalization liver parameters $>5$ ULN. ${ }^{5}$ A Chinese study reported that a significantly higher proportion of patients with abnormal liver function (57.8\%) had received Lopinavir/Ritonavir after admission compared to patients with normal liver function $(31.3 \%)^{7}$ Another Chinese study reported that the use of Lopinavir/ Ritonavir \pm Ribavirin + interferon beta (OR 1.94, $\mathrm{p}=0.006)$ was independently associated with ALT/AST elevation. ${ }^{20}$ Based on previous studies and our results, we suggested Hydroxychloroquine, Lopinavir/Ritonavir, and TCM should be used with caution in patients with abnormal ALT and LDH at hospital admission.

In a Chinese cohort of 675 patients with COVID-19, compared to patients with normal AST levels, mortality and risk of mechanical ventilation significantly increased 19.27-fold and 116.72-fold, respectively, in patients with AST above 3 -fold ULN. ${ }^{21}$ In another Chinese cohort, Cai et al found that the presence of abnormal liver tests and liver injury were associated with the progression to severe COVID-19. ${ }^{6}$ In a large Hong Kong cohort of 1040 COVID-19 patients, Yip et al found ALT/AST elevation and acute liver injury are independently associated with adverse clinical outcomes including admission to intensive care unit, use of invasive mechanical ventilation and/or death in COVID-19 patients. $^{20}$ Saini et al retrospectively analysed liver function tests of 170 patients with confirmed COVID-19, and also found number of patients with raised levels of any of the liver enzymes were 89 (58.5\%), out of which $43(48.31 \%)$ had liver injury, which manifested as increased severity in terms of ICU requirement $(p=0.0005) .^{22}$ In this study, abnormal liver parameters during hospitalization are associated with illness severity and mortality of COVID-19, with the strongest associations observed between peak liver tests and severe COVID-19, as well as peak liver tests and death. Based on previous studies and our results, we suggested monitoring levels of liver function parameters, which could assist in the optimum management of patients with COVID-19.

Many TCM were used in patients with COVID-19 in our cohort; therefore, the effect of TCM on liver functions should not be neglected in COVID-19 patients. $^{23}$ In fact, the TCM-related liver injury is not uncommon in patients with COVID-19. ${ }^{24}$ A meta-analysis showed that the TCM as a complementary therapy for treating COVID-19 may not be beneficial for improving liver function based on the current evidence. ${ }^{23}$ In this study, we found that the TCM use is one of the predictors of peak hospitalization ALT > 5 ULN. Based on previous studies and our results, we suggested that prevention and management of TCMinduced liver injury should be concerned in COVID-19 patients who received TCM therapy.

Besides liver injury, other gastrointestinal manifestations were also concerned in COVID-19 patients. At the age of COVID-19 crisis, gastrointestinal physicians may face rare gastrointestinal symptoms such as dysentery, pure hyperbilirubinemia, and so on. For example, Hormati et al have reported the clinical data in details as well as the result of chest CT of a COVID-19 patient with dysentery. ${ }^{25}$ In a case series, Hormati et al also have reported pure hyperbilirubinemia may be considered as rare gastrointestinal symptom of COVID-19. ${ }^{26}$ Therefore, it is necessary that all gastrointestinal physicians should be aware of the possible occurrence of these gastrointestinal symptoms (hepatic involvement, pure hyperbilirubinemia, dysentery) as an important prognosis of COVID-19 pneumonia and it should be exactly addressed in new referred patients to gastrointestinal clinic. In addition, Hormati et al address preventive strategies that may significantly reduce close contact between patients and gastrointestinal physicians for successful control of COVID-19 infection. $^{27}$ Preventive strategies should be performed to prevent transmission of COVID-19 infection from infected patients to uninfected gastrointestinal physicians and staff members during the performance of high-risk procedures. ${ }^{27}$

This study has several limitations. First, retrospective observational cohort study design with inclusion restricted to patients who were hospitalized within a single hospital, and limited access to laboratory, and medication variables, which may influence clinical outcomes. Second, this study did not elucidate the etiology of liver function test elevations in hospitalized patients with COVID-19. However, based on previous studies, we have reasons to believe that the drug's effects, possible viral inclusion in liver cells, systemic inflammation, and hypoxia are potential causes of liver injury in patients with COVID-19. ${ }^{28}$ Third, in our hospital, the qualitative analysis (positive or negative) of SARS-CoV-2 RNA is used to guide the diagnosis and treatment of COVID19 patients. Although CT (cycle-threshold)-value for viral load can support in the better interpretation of clinical decisions, in this retrospective study, the quantification of SARS$\mathrm{CoV}-2$ viral load is not available. 
In conclusion, in this large sample retrospective cohort study, we described the longitudinal changes of liver function parameters in patients with COVID-19. In addition, we confirmed patients with abnormal liver function parameters were at increased risk of severe COVID-19 and death. The COVID19-related liver injury is related to antiviral medication use.

\section{Ethics Approval and Consent to Participate}

Although this is a retrospective study, at hospital admission, all patients provided verbal consent for their clinical data might be used for further medical study. Shanghai Public Health Clinical Center Ethics Committee approved this study, including the verbal informed consent process. When we performed the study, all personal information of patients was de-identified to protect privacy. The procedures followed were in accordance with the ethical standards of the Helsinki Declaration (1964, amended most recently in 2008) of the World Medical Association.

\section{Consent for Publication}

All authors read and approved the manuscript.

\section{Acknowledgments}

We thank all doctors who work in Shanghai Public Health Clinical Center for their efforts in the diagnosis and treatment of patients with COVID-19.

\section{Author Contributions}

All authors made a significant contribution to the work reported, whether that is in the conception, study design, execution, acquisition of data, analysis and interpretation, or in all these areas; took part in drafting, revising or critically reviewing the article; gave final approval of the version to be published; have agreed on the journal to which the article has been submitted; and agree to be accountable for all aspects of the work.

\section{Role of the Sponsor}

The funding organization is a public institution and had no role in the design and conduct of the study; collection, management, and analysis of the data; or preparation, review, and approval of the manuscript.

\section{Funding}

This study was supported by grant No. 19YF1441200 from Shanghai Sailing Plan Program.

\section{Disclosure}

The authors reported no conflicts of interest for this work.

\section{References}

1. Wu F, Zhao S, Yu B, et al. A new coronavirus associated with human respiratory disease in China. Nature. 2020;579(7798):265-269. doi:10.1038/s41586-020-2008-3

2. Awadasseid A, Wu Y, Tanaka Y, et al. Initial success in the identification and management of the coronavirus disease 2019 (COVID-19) indicates human-to-human transmission in Wuhan, China. Int J Biol Sci. 2020;16:1846-1860. doi:10.7150/ijbs. 45018

3. Weekly operational update on COVID-19. Available from: https:// www.who.int/publications/m/item/weekly-epidemiological-update. Accessed January 19, 2021.

4. Hoffmann M, Kleine-Weber H, Schroeder S, et al. SARS-CoV-2 cell entry depends on ACE2 and TMPRSS2 and is blocked by a clinically proven protease inhibitor. Cell. 2020;181:271-280.e8. doi:10.1016/j. cell.2020.02.052

5. Hundt MA, Deng Y, Ciarleglio MM, et al. Abnormal liver tests in COVID-19: a retrospective observational cohort study of 1827 patients in a major U.S. hospital network. Hepatology. 2020;72 (4):1169-1176. doi:10.1002/hep.31487

6. Cai Q, Huang D, Yu H, et al. COVID-19: abnormal liver function tests. J Hepatol. 2020;73:566-574. doi:10.1016/j.jhep.2020.04.006

7. Fan Z, Chen L, Li J, et al. Clinical features of COVID-19-related liver functional abnormality. Clin Gastroenterol Hepatol. 2020;18:1561-1566. doi:10.1016/j.cgh.2020.04.002

8. Zhang Y, Zheng L, Liu L, et al. Liver impairment in COVID-19 patients: a retrospective analysis of 115 cases from a single centre in Wuhan city, China. Liver Int. 2020;40:2095-2103. doi:10.1111/liv.14455

9. Lei F, Liu YM, Zhou F, et al. Longitudinal association between markers of liver injury and mortality in COVID-19 in China. Hepatology. 2020;72:389-398. doi:10.1002/hep.31301

10. National Health Commision of the People's Republic of China. New coronavirus pneumonia prevention and control program (the fifth edition).. 2020. Available from: http://www.nhc.gov.cn/yzygj/ s7653p/202002/3b09b894ac9b4204a79db5b8912d4440.shtml. Accessed February 5, 2020.

11. Rich JT, Neely JG, Paniello RC, et al. A practical guide to understanding Kaplan-Meier curves. Otolaryngol Head Neck Surg. 2010;143:331-336. doi:10.1016/j.otohns.2010.05.007

12. Gupta A, Madhavan MV, Sehgal K, et al. Extrapulmonary manifestations of COVID-19. Nat Med. 2020;26:1017-1032. doi:10.1038/ s41591-020-0968-3

13. Sultan S, Altayar O, Siddique SM, et al. AGA Institute rapid review of the gastrointestinal and liver manifestations of COVID-19, meta-analysis of international data, and recommendations for the consultative management of patients with COVID-19. Gastroenterology. 2020;159:320-334.e27. doi:10.1053/j.gastro.2020.05.001

14. Cholankeril G, Podboy A, Aivaliotis VI, et al. High prevalence of concurrent gastrointestinal manifestations in patients with severe acute respiratory syndrome Coronavirus 2: early experience from California. Gastroenterology. 2020;159(2):775-777. doi:10.1053/j. gastro.2020.04.008

15. Hajifathalian K, Krisko T, Mehta A, et al. Gastrointestinal and hepatic manifestations of 2019 novel coronavirus disease in a large cohort of infected patients from New York: clinical implications. Gastroenterology. 2020;159:1137-1140.e2. doi:10.1053/j.gastro.20 20.05.010

16. Singh S, Khan A. Clinical characteristics and outcomes of coronavirus disease 2019 among patients with preexisting liver disease in the United States: a Multicenter Research Network Study. Gastroenterology. 2020;159:768-771.e3. 
17. Phipps MM, Barraza LH, LaSota ED, et al. Acute liver injury in COVID-19: prevalence and association with clinical outcomes in a large US cohort. Hepatology. 2020;72(3):807-817. doi:10.1002/hep.31404

18. Pirola CJ, Sookoian S. COVID-19 and ACE2 in the liver and gastrointestinal tract: putative biological explanations of sexual dimorphism. Gastroenterology. 2020;159:1620-1621. doi:10.1053/j. gastro.2020.04.050

19. Jothimani D, Venugopal R, Abedin MF, et al. COVID-19 and the liver. J Hepatol. 2020;73:1231-1240. doi:10.1016/j.jhep.2020.06.006

20. Yip TC, Lui GC, Wong VW, et al. Liver injury is independently associated with adverse clinical outcomes in patients with COVID-19. Gut. 2020;70 (4):733-742. doi:10.1136/gutjnl-2020-321726

21. Huang $\mathrm{H}$, Chen $\mathrm{S}, \mathrm{Li} \mathrm{H}$, et al. The association between markers of liver injury and clinical outcomes in patients with COVID-19 in Wuhan. Aliment Pharmacol Ther. 2020. doi:10.1111/apt.15962

22. Saini RK, Saini N, Ram S, et al. COVID-19 associated variations in liver function parameters: a retrospective study. Postgrad Med J. 2020. doi:10.1136/postgradmedj-2020-138930

23. Shi S, Wang F, Li J, et al. The effect of Chinese herbal medicine on digestive system and liver functions should not be neglected in COVID-19: an updated systematic review and meta-analysis. IUBMB Life. 2021. doi:10.1002/iub.2467
24. Mao R, Qiu Y, He JS, et al. Manifestations and prognosis of gastrointestinal and liver involvement in patients with COVID-19: a systematic review and meta-analysis. Lancet Gastroenterol Hepatol. 2020;5:667-678. doi:10.1016/S24681253(20)30126-6

25. Hormati A, Ghadir MR, Saeidi M, et al. Dysentery as a rare GI symptom found in COVID-19 patients. Gastroenterol Hepatol. 2021;44:31-34. doi:10.1016/j.gastrohep.2020.06.005

26. Hormati A, Ghadir MR, Saeidi M, et al. Hepatic involvement as hyperbilirubinemia in patients with COVID-19: case series from Iran. Infect Disord Drug Targets. 2021;21:. doi:10.2174/ 1871526521666210218201601

27. Hormati A, Ghadir MR, Zamani F, et al. Preventive strategies used by GI physicians during the COVID-19 pandemic. New Microbes New Infect. 2020;35:100676. doi:10.1016/j.nmni.2020.100676

28. Ali N, Hossain K. Liver injury in severe COVID-19 infection: current insights and challenges. Expert Rev Gastroenterol Hepatol. 2020;14:879-884. doi:10.1080/17474124.2020.1794812
Clinical Interventions in Aging

\section{Publish your work in this journal}

Clinical Interventions in Aging is an international, peer-reviewed journal focusing on evidence-based reports on the value or lack thereof of treatments intended to prevent or delay the onset of maladaptive correlates of aging in human beings. This journal is indexed on PubMed Central, MedLine, CAS, Scopus and the Elsevie
Dovepress

Bibliographic databases. The manuscript management system is completely online and includes a very quick and fair peer-review system, which is all easy to use. Visit http://www.dovepress.com/ testimonials.php to read real quotes from published authors. 